\title{
The Sodium-Driven Chloride/Bicarbonate Exchanger in Presynaptic Terminals
}

\author{
Alain C. Burette ${ }^{1,}$, Richard J. Weinberg ${ }^{1,2}$, Patrick Sassani $^{3}$, Natalia Abuladze $^{3}$, Liyo Kao ${ }^{3}$, \\ and Ira Kurtz 3,4 \\ ${ }^{1}$ Department of Cell and Developmental Biology, University of North Carolina, Chapel Hill, North \\ Carolina 27599 \\ ${ }^{2}$ Neuroscience Center, University of North Carolina, Chapel Hill, North Carolina 27599 \\ ${ }^{3}$ David Geffen School of Medicine, University of California-Los Angeles, Los Angeles, California \\ 90095
}

${ }^{4}$ Brain Research Institute, University of California-Los Angeles, Los Angeles, California 90095

\begin{abstract}
The sodium-driven chloride/bicarbonate exchanger (NDCBE), a member of the SLC4 family of bicarbonate transporters, was recently found to modulate excitatory neurotransmission in hippocampus. By using light and electron microscopic immunohistochemistry, we demonstrate here that NDCBE is expressed throughout the adult rat brain, and selectively concentrates in presynaptic terminals, where it is closely associated with synaptic vesicles. NDCBE is in most glutamatergic axon terminals, and is also present in the terminals of parvalbumin-positive $\gamma$ aminobutyric acid (GABA)ergic cells. These findings suggest that NDCBE can regulate glutamatergic transmission throughout the brain, and point to a role for NDCBE as a possible regulator of GABAergic neurotransmission.
\end{abstract}

\section{Keywords}

synaptic vesicle; glutamate; GABA; pH; VGLUT; VGAT; SLC4A8

\begin{abstract}
Vesicular release of neurotransmitter is triggered by calcium entry through voltagedependent calcium channels. Modulation of transmitter release, an important component of synaptic plasticity, may be achieved via changes in release probability (Stevens, 2004).

However, release may also be modulated by changing the concentration of neurotransmitter in presynaptic vesicles (Edwards, 2007; Lisman et al., 2007; Wilson et al., 2005). Glutamate and $\gamma$-aminobutyric acid (GABA) are loaded into neurosecretory vesicles by specific transporters driven by a vacuolar proton pump, whose activity establishes an electrochemical gradient across the vesicular membrane, with the inside of the vesicle being positive and acidic (Cidon et al., 1983). The glutamate transporters responsible for loading glutamate into vesicles (VGLUT1-3; Bellocchio et al., 2000; Takamori et al., 2000) are driven by the electrical component $(\Delta \Psi)$ of the gradient generated by the vacuolar proton pump (Edwards, 2007; Maycox et al., 1988), whereas vesicular GABA transporter/vesicular inhibitory amino acid transporter (VGAT/VIAAT), which is responsible for loading GABA and glycine, depends on both $\Delta \Psi$ and $\Delta \mathrm{pH}$ (Hell et al., 1990; Kish et al., 1989).
\end{abstract}

C 2011 Wiley Periodicals, Inc.

"CORRESPONDENCE TO: Alain C. Burette, Dept. of Cell \& Developmental Biology, University of North Carolina, CB \# 7090, Chapel Hill, NC 27599. alain.burette@gmail.com.. 
The uptake of glutamate (via VGLUT1-3) and of GABA/glycine (via VGAT) into synaptic vesicles is coupled to chloride. It appears that VGAT can co-transport $\mathrm{Cl}^{-}$and GABA in a $\Delta \Psi$-dependent manner (Juge et al., 2009). Likewise, Schenck et al. (2009) reported that VGLUT itself can act as a chloride transporter, thus acting as a glutamate/chloride exchanger (Schenck et al., 2009). However, this result is controversial; Juge et al. (2010) concluded instead that VGLUT is allosterically activated by $\mathrm{Cl}^{-}$. Although the underlying mechanisms remain controversial, these studies and others point to chloride as a key regulator of vesicular storage of both GABA and glutamate, and show that $\left[\mathrm{Cl}^{-}\right]$determines both the speed and the extent of neurotransmitter loading.

CIC-3, a member of the chloride channel/transporter family, has been a prime candidate for mediation of the translocation of $\mathrm{Cl}^{-}$in synaptic vesicles necessary for transmitter loading (Maritzen et al., 2008; Stobrawa et al., 2001; Wang et al., 2006). However, the sodiumdriven chloride/bicarbonate exchanger NDCBE (encoded by the SLC4A8 gene; Grichtchenko et al., 2001; Parker et al., 2008) might also regulate vesicular $\left[\mathrm{Cl}^{-}\right]$. One proteomic study found NDCBE associated with the synaptic vesicle fraction (Phillips et al., 2005). Consistent with this result, a recent report finds NDCBE in excitatory synaptic terminals within the hippocampus, and demonstrates that its disruption impairs glutamate release from hippocampal neurons (Sinning et al., 2011).

Here we investigate the regional, cellular, and subcellular localization of NDCBE in the adult rat brain. We show that NDCBE is expressed throughout the brain, and selectively concentrates in presynaptic terminals, where it is closely associated with synaptic vesicles. NDCBE was found in most glutamatergic axon terminals, and was also present in the terminals of parvalbumin-positive GABAergic cells. These findings suggest that NDCBE can regulate glutamatergic transmission throughout the brain, and also point to a possible role for NDCBE as a regulator of GABAergic neurotransmission.

\section{MATERIALS AND METHODS}

All procedures related to the care and treatment of animals were in accordance with institutional and NIH guidelines; all animals use protocols have been reviewed and approved by the relevant Institutional Animal Care and Use Committee.

\section{Primary antibodies}

Table 1 lists the primary antibodies used in this study, which were as follows:

1. NDCBE-1r. NCDBE is expressed as at least three splice variants, NDCBE-A, -B, and -C (Parker et al., 2008). We generated a polyclonal rabbit antibody by immunizing rabbits with a synthetic peptide (LSINSGNTKEKSPFN, corresponding to the C-terminal fragment of rat NDCBE-A/C), coupled to keyhole limpet hemocyanin (KLH). The specificity of this antibody (NDCBE-1r) was verified by using HEK293 cells transfected with NDCBE-A using Lipofectamine (Invitrogen, Carlsbad, CA), as described (Zhu et al., 2010). As expected, the antibody recognizes a single band of $\sim 130 \mathrm{kDa}$ on immunoblots from transfected cells (Fig. 1A); no band was observed by using untransfected cells. Furthermore, NDCBE-1r stained HEK293 cells transfected with NDCBE-A (Fig. 1B), but not untransfected cells. A single band of $\sim 135 \mathrm{kDa}$ was also observed on immunoblots from rat brain lysate (Fig. 1A). Preadsorption of the antibody with the corresponding antigenic peptide eliminated the immunoreactivity for both Western blots (Fig. 1A) and immunohistochemistry (Fig. 1 C,D).

2. VGAT. For VGAT, a mouse monoclonal anti-VGAT antibody (clone 117G4, Synaptic Systems) was used. This antibody was raised against a synthetic peptide 
AEPPVEGDIHYQR (amino acids 75-87 in rat), coupled to KLH via an added Nterminal cysteine. This antibody recognizes a single band of the expected molecular weight ( $\sim 57 \mathrm{kDa}$ ) for VGAT in Western blots of lysates from mouse brain and retina (Guo et al., 2009). Furthermore, the staining pattern of this antibody is nearly identical to that reported using different VGAT antibodies (Boulland et al., 2009; Bragina et al., 2010; Chaudhry et al., 1998).

3. VGLUT1. For VGLUT1, a guinea pig polyclonal antibody (Chemicon, Temecula, CA; AB5905, lot 24041061) raised against rat VGLUT1 was used. We previously sequenced the immunogenic peptide (Chemicon, AG208) and concluded that this antibody was raised against a C-terminal peptide (GATHSTVQPPRPPPPVRDY; Melone et al., 2005). The antibody recognizes a single band of $\sim 60 \mathrm{KDa}$ on immunoblots of synaptic membrane fractions from rat cerebral cortex. Furthermore, immunogold labeling shows that VGLUT1 immunoreactivity is selectively associated with axon terminals forming asymmetric synapses in cerebral cortex and hippocampus.

4. Parvalbumin. For parvalbumin, a monoclonal anti-parvalbumin antibody (Sigma, St. Louis, MO: PARV-19, P-3088) was used. This antibody was derived from a hybridoma produced by fusing mouse myeloma cells with splenocytes from a mouse immunized with purified frog muscle parvalbumin. We previously controlled for specificity by performing immunohistochemistry on tissue from parvalbumin-knockout and corresponding control mice (Burette et al., 2009). No staining was observed in mice lacking parvalbumin.

\section{Tissue preparation}

After deep anesthesia was induced with sodium pentobarbital (60 mg/kg, i.p.), male Sprague-Dawley rats (200-350 g, Charles River, Raleigh, NC) were intracardially perfused with $500 \mathrm{ml}$ of fixative: either $4 \%$ freshly depolymerized paraformaldehyde in phosphate buffer (PB; 0.1 M, pH 7.4), for light microscopy (LM); or a mixture of $4 \%$ paraformaldehyde and $0.1 \%$ glutaraldehyde in $\mathrm{PB}$, for electron microscopy (EM). Brains were sectioned at $40-60 \mu \mathrm{m}$ on a Vibratome and collected in cold PB.

\section{Light microscopy}

Free-floating sections were incubated in $10 \%$ normal donkey serum (NDS) and then in primary antibody (NDCBE-1r, 1:2,000). Antigenic sites were visualized by donkey IgG conjugated to DyLight 488 (1:200; Jackson ImmunoResearch; West Grove, PA). For double labeling with parvalbumin, monoclonal anti-parvalbumin antibody (1:40,000, Sigma, PARV-19, P-3088) was then applied overnight and visualized by a condary antibody conjugated to DyLight 549 (1:200, Jackson ImmunoResearch).

For triple labeling, a second primary antibody (VGLUT1, 1:5,000, guinea pig antiVGLUT1, AB5905, lot 23080329, Chemicon) and a third primary antibody (VGAT, 1:1,000, monoclonal mouse antibody, clone 117G4, Synaptic Systems, Göttingen, Germany) were then applied overnight and visualized by secondary antibodies conjugated to DyLight 549 and Cy5 (1:200, Jackson ImmunoResearch). Control experiments, in which the primary antibodies were omitted, were performed to control for nonspecific binding of the secondary antibody. Sections were examined with a Leica SP2 confocal microscope.

\section{Electron microscopy}

Floating sections treated with $1 \%$ sodium borohydride were blocked with $20 \%$ NDS, and incubated in primary antibody (NDCBE-1C, 1:2,000). Sections were then incubated with biotinylated goat-anti rabbit IgG (1:200, Jackson ImmunoResearch), followed by 1.4-nm 
gold particles conjugated to streptavidin (1:50; Nanoprobes, Yaphank, NY). Sections were washed in $0.05 \mathrm{M} \mathrm{Na}$ acetate (to remove phosphate and chloride ions), followed by silver enhancement with IntenSE-M (Amersham Biosciences, Arlington Heights, IL). Sections were then postfixed in $0.5-1 \%$ osmium tetroxide in $0.1 \mathrm{M}$ PB for $35-45$ minutes and incubated with $1 \%$ uranyl acetate in maleate buffer $(0.1 \mathrm{M}, \mathrm{pH}$ 6.0) for 1 hour. After dehydration, sections were infiltrated with a mixture of Epon and Spurr resins (Electron Microscopy Sciences, Hatfield, PA) and flat-mounted between sheets of ACLAR fluoropolymer (Electron Microscopy Sciences) within glass slides. Seventy-nanometer sections were cut, mounted on 200 mesh copper grids, contrasted with uranyl acetate and Sato's lead, and examined in a Philips Tecnai electron microscope (Hillsboro, OR) at $80 \mathrm{kV}$.

\section{Data analysis and preparation of plates}

To test whether staining for two antigens was positively or negatively correlated, we performed intensity correlation analysis as described by Li et al. (2004) by using the Intensity Correlation Analysis plug-in in ImageJ (see the ImageJ website of the Wright Cell Imaging Facility, Toronto Western Research Institute, ON, Canada: www.uhnresearch.ca/ wcif). We used Corel Draw v.14 (Corel, Ottawa, ON, Canada) to sharpen images (via unsharp mask), adjust brightness and contrast, and compose final plates. These adjustments were done exclusively to enhance the presentation quality of figures, without altering the scientific data content of the images.

\section{RESULTS}

NDCBE was detected throughout the brain, concentrated in gray matter. The overall regional pattern of staining resembled that shown in previous studies in rodent (Chen et al., 2008) and human (Damkier et al., 2007). However, these reports emphasized a predominantly soma-dendritic pattern, whereas immunostaining with our antibody was concentrated in puncta within the neuropil, consistent with the results of Sinning et al. (2011). This might in part reflect technical differences or higher back-ground staining in previous studies, but other factors may be involved (see Discussion).

In the cerebral cortex, puncta immunopositive for NDCBE were found in the neuropil throughout all layers; immunoreactive cell bodies were not seen (Fig. 2A). NDCBE staining was moderate to intense throughout the hippocampal formation, and weaker in layers rich in somata, including the pyramidal cell layer of Ammon's horn and the granule cell layer of the dentate gyrus (Fig. 2B). The large mossy fiber boutons in the stratum lucidum of CA3 showed prominent NDCBE staining. High-magnification views of staining in the striatum showed a typical punctate organization (Fig. 2C,D). Punctate staining was also seen in the neuropil of the thalamus (Fig. 2E). In the cerebellar cortex, NDCBE staining was conspicuous in the molecular and granule cell layers (Fig. 2F). In the molecular layer, densely packed immunoreactive puncta surrounded Purkinje cell dendrites, which were themselves devoid of staining. In the granule cell layer, intensely stained glomeruli were scattered among unstained granule cells. Punctate staining for NDCBE was also seen throughout the brainstem (Fig. 2G).

To better characterize the nature of these puncta, we performed EM, using pre-embedding immunogold labeling followed by silver intensification to optimize sensitivity. Gold/silver particles coding for NDCBE labeled numerous presynaptic terminals throughout the brain (Figs. 3, 4). Labeling was seen in a large fraction of presynaptic terminals in the cerebral cortex (Fig. 3A-C), hippocampus (Fig. 3D-F), cerebellar cortex (Fig. 4A-C), and brainstem (Fig. 4D,E). Immunolabeling in the large terminals of mossy fibers in the hippocampus (Fig. 3E,F), and over vesicle-rich areas within the central presynaptic terminal of synaptic glomeruli in cerebellar cortex (Fig. 4C), was consistent with our LM results. NDCBE 
labeling was found in asymmetric (presumably glutamatergic) synapses, but could also be detected in symmetric synapses (Fig. 3A). In contrast to the prominent presynaptic labeling, little or no labeling was seen on the postsynaptic side. Occasional particles representing NDCBE were seen in axons. Although NDCBE was concentrated in axon terminals, it showed no obvious association with the plasma membrane, but instead concentrated selectively over presynaptic vesicles (Fig. 4).

To assess the extent of NDCBE expression in axon terminals, we performed triple-labeling experiments for NDCBE with markers for excitatory and inhibitory vesicles. Throughout the brain, visual inspection suggested marked co-localization of NDCBE with both VGLUT1 and VGAT staining (Figs. 5, 6). We performed quantitative analysis of images from cerebral cortex to assess the extent of this co-localization. Analysis of 35 random fields in layer $\mathrm{V}$ of cerebral cortex showed that $90 \pm 4 \%$ of VGLUT1-positive puncta $(n=1,295)$ were also positive for NDCBE. In contrast, NDCBE was found in a smaller fraction of VGAT-positive puncta $(61 \pm 9, \mathrm{n}=405)$.

In the cerebral cortex and hippocampus, NDCBE/VGAT-positive puncta could often be seen surrounding immunonegative somata, reminiscent of the basket terminals of fast-spiking interneurons (Fig. 6A-D). To test this hypothesis, we performed double-labeling for NDCBE and parvalbumin, demonstrating co-localization in puncta surrounding somata in the cortex and hippocampus (Fig. 7A-F). Co-localization was also seen in the thalamus, but it was found in scattered puncta through the neuropil instead of being organized into pericellular baskets (Fig. 7G-I). Although we were unable to examine all possible markers for inhibitory neurons, it was noteworthy that material double-labeled for NDCBE and somatostatin (a marker for regular-spiking interneurons) failed to show any sign of co-localization (data not shown).

If NDCBE were an integral component of synaptic vesicles, one would predict that the intensity of staining for NDCBE should covary with that for VGLUT and VGAT. To test this hypothesis, raw confocal images were analyzed by using intensity correlation analysis as described by Li et al. (2004). Figure 8A-D illustrates results from this technique, by using the raw image used for Figure 6A-D. (Note that in Fig. 6A-D, the raw image was cropped, and contrast and brightness adjusted to improve visibility.) Intensity correlation quotients (ICQs; computed such that -0.5 represents perfect negative covariance, and 0.5 represents perfect positive covariance between two antigens) were consistently strongly positive (average ICQ $0.35 \pm 0.03$ for NDCBE/VGLUT, and $0.27 \pm 0.07$ for NDCBE/VGAT, $\mathrm{n}=60$ fields, 10 each from the cerebral cortex, hippocampus, striatum, cerebellum, cochlear nucleus, and thalamus.

These data consider covariation of pixels throughout the microscopic fields. To assess whether the two channels covaried when attention was restricted to presynaptic terminals, we examined a sample of 692 NDCBE-positive puncta also immunopositive for either VGLUT1 or VGAT in the cerebral cortex. Scatterplots of mean intensity of NDCBE in a punctum against the corresponding VGLUT intensity show a strong positive correlation among excitatory terminals $\left(\mathrm{r}^{2}=0.76\right.$, Fig. $\left.8 \mathrm{E}\right)$; likewise, a strong positive correlation was seen among inhibitory terminals $\left(r^{2}=0.86\right.$, Fig. $\left.8 \mathrm{~F}\right)$. As a control, we examined the relationship between VGLUT1 and VGAT in presumed synaptic puncta and found no significant relationship $\left(r^{2}=.04\right.$, Fig. $\left.8 \mathrm{G}\right)$.

\section{DISCUSSION}

Even minor changes in $\mathrm{pH}$ within the brain can impact neurological function. $\mathrm{pH}$ modulates both intrinsic neuronal excitability (Chesler, 2003; Dulla et al., 2005; Jacobs et al., 2008) 
and synaptic transmission (Ahdut-Hacohen et al., 2004; DeVries, 2001; Dietrich and Morad, 2010; Makani and Chesler, 2007). Accordingly, the brain contains multiple acid/base transporters, including members of the SLC4 bicarbonate (carbonate) transporter family. One of these, NDCBE (SLC4A8), mediates $\mathrm{Na}^{+}$-driven $\mathrm{Cl}^{-} / \mathrm{HCO}_{3}^{-}$exchange (Grichtchenko et al., 2001; Parker et al., 2008). Similar $\mathrm{Na}^{+}$-driven $\mathrm{Cl}^{-}-\mathrm{HCO}_{3}^{-}$ exchangers have been shown to regulate $\mathrm{pH}$ in invertebrate neuronal systems, including molluscan neurons and the squid giant axon (Boron and De Weer, 1976; Thomas, 1977). However, recent work raises the possibility that NDCBE may influence neurotransmission independent of its effects on $\mathrm{pH}$ (Kim and Trussell, 2009).

NDCBE and NDCBE-like activity has been detected in multiple brain regions in both rodents and humans (Baxter and Church, 1996; Chen et al., 2008; Damkier et al., 2007; Schwiening and Boron, 1994). The present study reveals a highly selective targeting of NDCBE to axon terminals, where it is closely associated with synaptic vesicles. Because NDCBE is an integral membrane protein, we conclude that the large majority of the vesicleassociated pool must be inserted into the vesicle membrane. This selectivity for terminals is consistent with the results of Sinning et al. (2011), but at variance with Chen et al. (2008). We suspect this reflects minor technical issues, but this discrepancy might reflect differences in targeting of different NDCBE splice variants (Parker et al., 2008): The N-terminus antibodies used by Boron's group are likely to recognize the A and B splice variants, whereas the antibodies used by Sinning et al. (2011) and by us were raised against Cterminal peptides, and are therefore likely to recognize the $\mathrm{A}$ and $\mathrm{C}$ variants; thus, our data are consistent with the possibility that the B splice variant is targeted to the soma-dendritic compartment.

What might be the functional significance of NDCBE in the presynaptic terminal? The work of Sinning et al. demonstrates that this protein plays a role in exocytosis; our finding that NDCBE shows little association with the plasma membrane leads us to conclude that the observed effect must be via an action at the vesicular membrane itself. Sinning's results implicitly suggest that NDCBE may also influence other aspects of synaptic transmission; together with previous work, this leads us to speculate that NDCBE may play a role in transmitter uptake and storage.

Chloride ions are needed for acidification of synaptic vesicles, and recent evidence shows that $\mathrm{Cl}^{-}$can regulate both VGLUT and VGAT (Juge et al., 2009; Schenck et al., 2009). However, it has been difficult to identify the chloride channel or transporter responsible. CIC-3, a member of the chloride channel/transporter family, has been a prime candidate for mediation of the translocation of $\mathrm{Cl}^{-}$in synaptic vesicles necessary for neurotransmitter loading (Maritzen et al., 2008; Riazanski et al., 2011; Stobrawa et al., 2001; Wang et al., 2006). Riazanski and collaborators (2011) recently demonstrated that CIC-3 modulates inhibitory synaptic strength by altering the magnitude of acidification in GABAergic vesicles, thereby decreasing quantal size. In contrast, loss of ClC-3 had little effect on acidification of glutamate-containing vesicles, consistent with previous evidence for only modest changes in vesicular glutamate transport in CIC-3 knockout mice (Stobrawa et al., 2001). Moreover, isolated vesicles retained a biphasic dependence on $\mathrm{Cl}^{-}$even in the absence of ClC-3, implying that another protein must regulate vesicular $\left[\mathrm{Cl}^{-}\right]$(Stobrawa et al., 2001). Our data showing that NDCBE is tightly linked to synaptic vesicles lead us to propose that NDCBE might play this role.

We also found NDCBE in a subpopulation of GABAgergic presynaptic terminals, suggesting a role in GABAergic transmission. Our data show that parvalbumin-positive basket terminals express especially high levels of NDCBE. These terminals arise from a subpopulation of interneurons remarkable for their capacity to fire sustained high-frequency 
trains of action potentials. Accordingly, we speculate that NDCBE helps to supplement CIC-3 in these terminals to permit effective transmitter reloading during sustained highfrequency firing.

\section{CONCLUSIONS}

The targeting of NDCBE to presynaptic vesicles is prominent throughout the brain. This phenomenon, which is seen in the large majority of excitatory synapses and in an important subpopulation of inhibitory synapses, points to a role for NDCBE in regulation of neurotransmitter release. Although the mechanistic details remain to be established, this finding is especially intriguing because NDCBE couples $\mathrm{Cl}^{-}$transport to $\mathrm{pH}$ (via $\mathrm{HCO}_{3}^{-}$), to ongoing metabolic activity (via cellular $\mathrm{CO}_{2}$ and $\mathrm{H}^{+}$production), and to ongoing synaptic activity (via intracellular $\left[\mathrm{Na}^{+}\right]$). Thus, NDCBE is well positioned to integrate multiple signals to modulate neurotransmitter release.

\section{Acknowledgments}

We thank Kristen Phend and Susan Burette for histological assistance.

Grant sponsor: National Institutes of Health; Grant numbers: DK077162 and DK058563 (to I.K.); Grant number: NS35527 and NS39444 (to R.J.W.).

\section{LITERATURE CITED}

Ahdut-Hacohen R, Duridanova D, Meiri H, Rahamimoff R. Hydrogen ions control synaptic vesicle ion channel activity in Torpedo electromotor neurones. J Physiol. 2004; 556:347-352. [PubMed: 14978200]

Baxter KA, Church J. Characterization of acid extrusion mechanisms in cultured fetal rat hippocampal neurones. J Physiol. 1996; 493:457-470. [PubMed: 8782109]

Bellocchio EE, Reimer RJ, Fremeau RT Jr, Edwards RH. Uptake of glutamate into synaptic vesicles by an inorganic phosphate transporter. Science. 2000; 289:957-960. [PubMed: 10938000]

Boron WF, De Weer P. Intracellular $\mathrm{pH}$ transients in squid giant axons caused by $\mathrm{CO}_{2}, \mathrm{NH}_{3}$, and metabolic inhibitors. J Gen Physiol. 1976; 67:91-112. [PubMed: 1460]

Boulland JL, Jenstad M, Boekel AJ, Wouterlood FG, Edwards RH, Storm-Mathisen J, Chaudhry FA. Vesicular glutamate and GABA transporters sort to distinct sets of vesicles in a population of presynaptic terminals. Cereb Cortex. 2009; 19:241-248. [PubMed: 18502731]

Bragina L, Giovedi S, Barbaresi P, Benfenati F, Conti F. Heterogeneity of glutamatergic and GABAergic release machinery in cerebral cortex: analysis of synaptogyrin, vesicle-associated membrane protein, and syntaxin. Neuroscience. 2010; 165:934-943. [PubMed: 19909789]

Burette AC, Strehler EE, Weinberg RJ. "Fast" plasma membrane calcium pump PMCA2a concentrates in GABAergic terminals in the adult rat brain. J Comp Neurol. 2009; 512:500-513. [PubMed: 19025983]

Chaudhry FA, Reimer RJ, Bellocchio EE, Danbolt NC, Osen KK, Edwards RH, Storm-Mathisen J. The vesicular GABA transporter, VGAT, localizes to synaptic vesicles in sets of glycinergic as well as GABAergic neurons. J Neurosci. 1998; 18:9733-9750. [PubMed: 9822734]

Chen LM, Kelly ML, Parker MD, Bouyer P, Gill HS, Felie JM, Davis BA, Boron WF. Expression and localization of Na-driven $\mathrm{Cl}^{-} \mathrm{HCO}_{3}$ - exchanger (SLC4A8) in rodent CNS. Neuroscience. 2008; 153:162-174. [PubMed: 18359573]

Chesler M. Regulation and modulation of $\mathrm{pH}$ in the brain. Physiol Rev. 2003; 83:1183-1221. [PubMed: 14506304]

Cidon S, Ben-David H, Nelson N. ATP-driven proton fluxes across membranes of secretory organelles. J Biol Chem. 1983; 258:11684-11688. [PubMed: 6619137] 
Damkier HH, Nielsen S, Praetorius J. Molecular expression of SLC4-derived $\mathrm{Na}^{+}$-dependent anion transporters in selected human tissues. Am J Physiol Regul Integr Comp Physiol. 2007; 293:R2136-2146. [PubMed: 17715183]

DeVries SH. Exocytosed protons feedback to suppress the $\mathrm{Ca}^{2+}$ current in mammalian cone photoreceptors. Neuron. 2001; 32:1107-1117. [PubMed: 11754841]

Dietrich CJ, Morad M. Synaptic acidification enhances GABAA signaling. J Neurosci. 2010; 30:16044-16052. [PubMed: 21106843]

Dulla CG, Dobelis P, Pearson T, Frenguelli BG, Staley KJ, Masino SA. Adenosine and ATP link $\mathrm{pCO}_{2}$ to cortical excitability via $\mathrm{pH}$. Neuron. 2005; 48:1011-1023. [PubMed: 16364904]

Edwards RH. The neurotransmitter cycle and quantal size. Neuron. 2007; 55:835-858. [PubMed: 17880890]

Grichtchenko, Choi I, Zhong X, Bray-Ward P, Russell JM, Boron WF. Cloning, characterization, and chromosomal mapping of a human electroneutral $\mathrm{Na}^{+}$-driven $\mathrm{Cl}^{-} \mathrm{HCO}_{3}$ exchanger. $\mathrm{J}$ Biol Chem. 2001; 276:8358-8363. [PubMed: 11133997]

Guo C, Stella SL Jr. Hirano AA, Brecha NC. Plasmalemmal and vesicular gamma-aminobutyric acid transporter expression in the developing mouse retina. J Comp Neurol. 2009; 512:6-26. [PubMed: 18975268]

Hell JW, Maycox PR, Jahn R. Energy dependence and functional reconstitution of the gammaaminobutyric acid carrier from synaptic vesicles. J Biol Chem. 1990; 265:2111-2117. [PubMed: 1688846]

Jacobs S, Ruusuvuori E, Sipila ST, Haapanen A, Damkier HH, Kurth I, Hentschke M, Schweizer M, Rudhard Y, Laatikainen LM, Tyynela J, Praetorius J, Voipio J, Hubner CA. Mice with targeted Slc4a10 gene disruption have small brain ventricles and show reduced neuronal excitability. Proc Natl Acad Sci U S A. 2008; 105:311-316. [PubMed: 18165320]

Juge N, Muroyama A, Hiasa M, Omote H, Moriyama Y. Vesicular inhibitory amino acid transporter is a Cl-/gamma-aminobutyrate co-transporter. J Biol Chem. 2009; 284:35073-35078. [PubMed: 19843525]

Kim Y, Trussell LO. Negative shift in the glycine reversal potential mediated by a $\mathrm{Ca}^{2+}$ - and $\mathrm{pH}-$ dependent mechanism in interneurons. J Neurosci. 2009; 29:11495-11510. [PubMed: 19759298]

Kish PE, Fischer-Bovenkerk C, Ueda T. Active transport of gamma-aminobutyric acid and glycine into synaptic vesicles. Proc Natl Acad Sci U S A. 1989; 86:3877-3881. [PubMed: 2566998]

Li Q, Lau A, Morris TJ, Guo L, Fordyce CB, Stanley EF. A syntaxin 1, Galpha(o), and N-type calcium channel complex at a presynaptic nerve terminal: analysis by quantitative immunocolocalization. $\mathrm{J}$ Neurosci. 2004; 24:4070-4081. [PubMed: 15102922]

Lisman JE, Raghavachari S, Tsien RW. The sequence of events that underlie quantal transmission at central glutamatergic synapses. Nat Rev Neurosci. 2007; 8:597-609. [PubMed: 17637801]

Makani S, Chesler M. Endogenous alkaline transients boost postsynaptic NMDA receptor responses in hippocampal CA1 pyramidal neurons. J Neurosci. 2007; 27:7438-7446. [PubMed: 17626204]

Maritzen T, Keating DJ, Neagoe I, Zdebik AA, Jentsch TJ. Role of the vesicular chloride transporter ClC-3 in neuroendocrine tissue. J Neurosci. 2008; 28:10587-10598. [PubMed: 18923035]

Maycox PR, Deckwerth T, Hell JW, Jahn R. Glutamate uptake by brain synaptic vesicles. Energy dependence of transport and functional reconstitution in proteoliposomes. J Biol Chem. 1988; 263:15423-15428. [PubMed: 2902091]

Melone M, Burette A, Weinberg RJ. Light microscopic identification and immunocytochemical characterization of glutamatergic synapses in brain sections. J Comp Neurol. 2005; 492:495-509. [PubMed: 16228991]

Parker MD, Bouyer P, Daly CM, Boron WF. Cloning and characterization of novel human SLC4A8 gene products encoding $\mathrm{Na}^{+}$-driven $\mathrm{Cl}-/ \mathrm{HCO} 3-$ exchanger variants NDCBE-A, -C, and -D. Physiol Genomics. 2008; 34:265-276. [PubMed: 18577713]

Phillips GR, Florens L, Tanaka H, Khaing ZZ, Fidler L, Yates JR 3rd, Colman DR. Proteomic comparison of two fractions derived from the transsynaptic scaffold. J Neurosci Res. 2005; 81:762-775. [PubMed: 16047384] 
Riazanski V, Deriy LV, Shevchenko PD, Le B, Gomez EA, Nelson DJ. Presynaptic CLC-3 determines quantal size of inhibitory transmission in the hippocampus. Nat Neurosci. 2011; 14:487-494. [PubMed: 21378974]

Schenck S, Wojcik SM, Brose N, Takamori S. A chloride conductance in VGLUT1 underlies maximal glutamate loading into synaptic vesicles. Nat Neurosci. 2009; 12:156-162. [PubMed: 19169251]

Schwiening CJ, Boron WF. Regulation of intracellular $\mathrm{pH}$ in pyramidal neurones from the rat hippocampus by $\mathrm{Na}^{+}$-dependent $\mathrm{Cl}-\mathrm{HCO} 3-$ exchange. J Physiol. 1994; 475:59-67. [PubMed: 8189393]

Sinning A, Liebmann L, Kougioumtzes A, Westermann M, Bruehl C, Hubner CA. Synaptic glutamate release is modulated by the $\mathrm{Na}^{+}$-driven $\mathrm{Cl}-/ \mathrm{HCO} 3-$ exchanger Slc4a8. J Neurosci. 2011; 31:7300-7311. [PubMed: 21593314]

Stevens CF. Presynaptic function. Curr Opin Neurobiol. 2004; 14:341-345. [PubMed: 15194114]

Stobrawa SM, Breiderhoff T, Takamori S, Engel D, Schweizer M, Zdebik AA, Bosl MR, Ruether K, Jahn H, Draguhn A, Jahn R, Jentsch TJ. Disruption of ClC-3, a chloride channel expressed on synaptic vesicles, leads to a loss of the hippocampus. Neuron. 2001; 29:185-196. [PubMed: 11182090]

Takamori S, Rhee JS, Rosenmund C, Jahn R. Identification of a vesicular glutamate transporter that defines a glutamatergic phenotype in neurons. Nature. 2000; 407:189-194. [PubMed: 11001057]

Thomas RC. The role of bicarbonate, chloride and sodium ions in the regulation of intracellular $\mathrm{pH}$ in snail neurones. J Physiol. 1977; 273:317-338. [PubMed: 23429]

Wang XQ, Deriy LV, Foss S, Huang P, Lamb FS, Kaetzel MA, Bindokas V, Marks JD, Nelson DJ. CLC-3 channels modulate excitatory synaptic transmission in hippocampal neurons. Neuron. 2006; 52:321-333. [PubMed: 17046694]

Wilson NR, Kang J, Hueske EV, Leung T, Varoqui H, Murnick JG, Erickson JD, Liu G. Presynaptic regulation of quantal size by the vesicular glutamate transporter VGLUT1. J Neurosci. 2005; 25:6221-6234. [PubMed: 15987952]

Zhu Q, Kao L, Azimov R, Newman D, Liu W, Pushkin A, Abuladze N, Kurtz I. Topological location and structural importance of the NBCe1-A residues mutated in proximal renal tubular acidosis. $\mathbf{J}$ Biol Chem. 2010; 285:13416-13426. [PubMed: 20197274] 

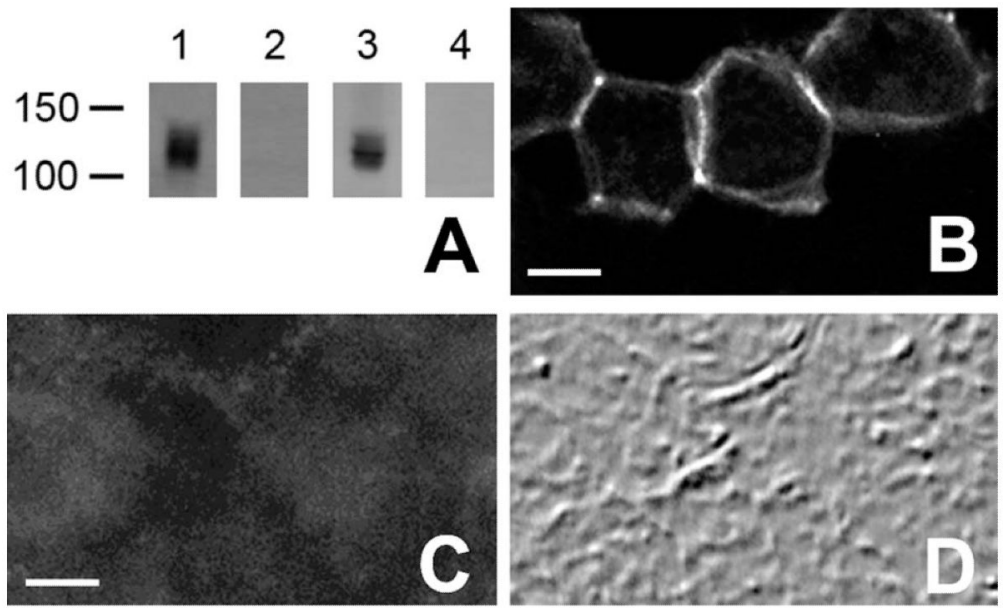

Figure 1.

A: Immunoblots, probed with the NDCBE-1C antibody: Lanes 1 and 2, brain lysate; Lanes 3 and 4 , lysate from HEK cells expressing NDCBE-A. Preadsorption of the antibody with the corresponding peptide (lanes 2 and 4) blocked the signal. B: Immunofluorescence labeling for NDCBE-1r in HEK 293 cells transfected with NDCBE-A. C:

Immunofluorescence labeling for NDCBE-1r in HEK 293 cells transfected with NDCBE-A in the presence of blocking peptide. D: Field in C, visualized by differential interference contrast imaging. Scale bar $=5 \mu \mathrm{m}$ in B (also applies to D) and C. 

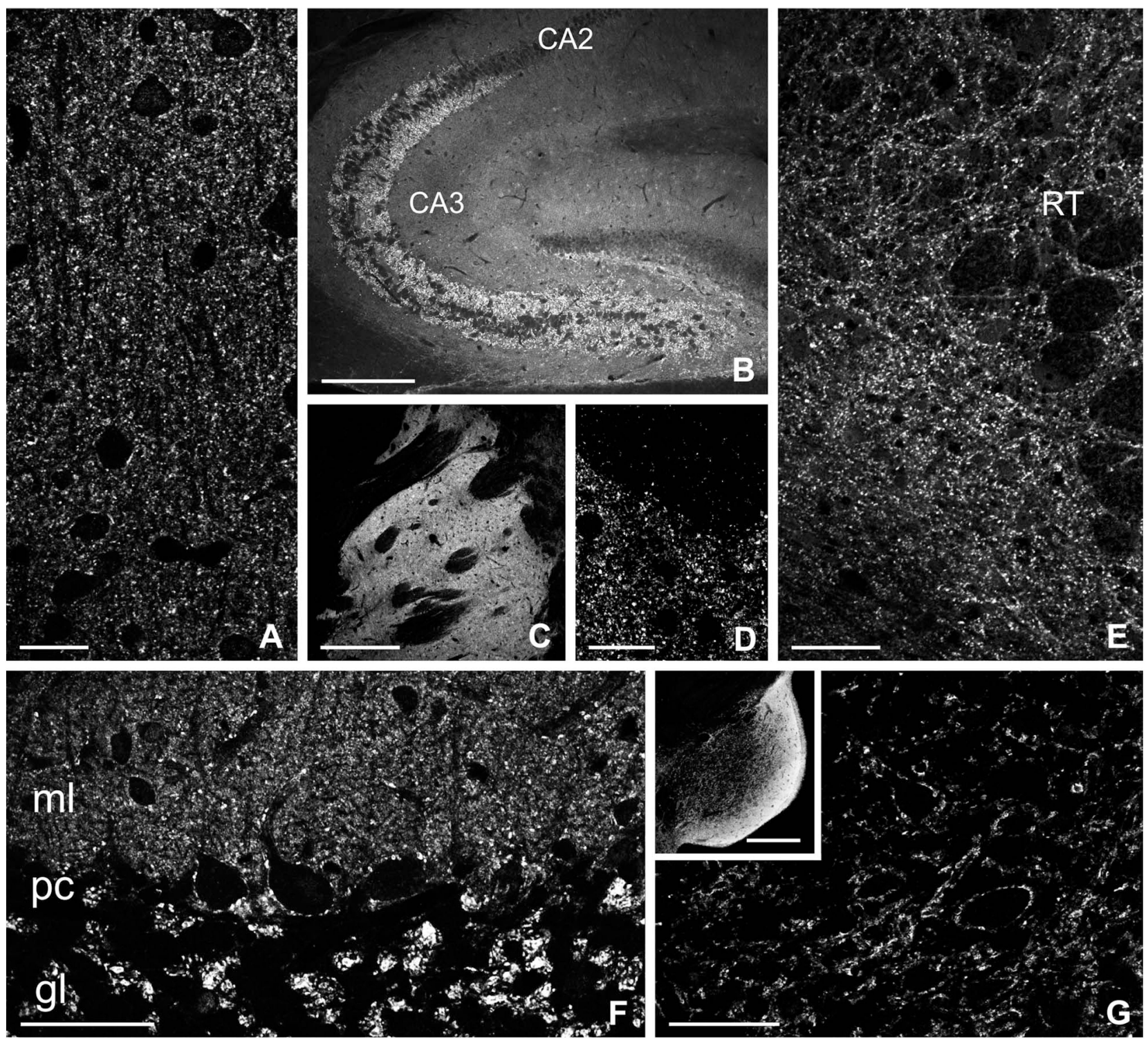

Figure 2.

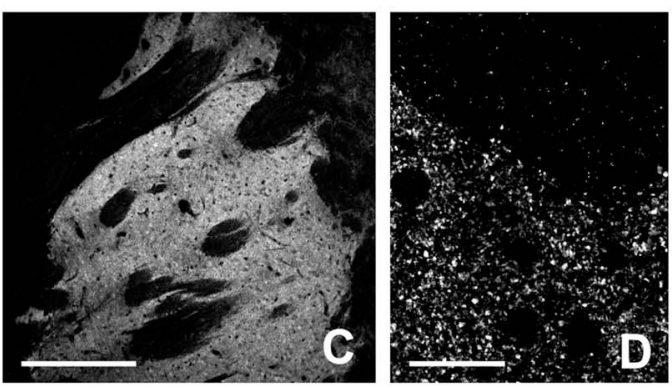

Immunofluorescence labeling for NDCBE in sections from rat brain. A: Cerebral cortex (S1, layer II). B: Hippocampus. C,D: Neostriatum. E: Thalamus. F: Cerebellar cortex. G: Cochlear nucleus. ml, molecular layer; pc, Purkinje cell layer; gl, granule cell layer, RT, reticular thalamic nucleus. Scale bar $=25 \mu \mathrm{m}$ in A,D; $300 \mu \mathrm{m}$ in B,C; $50 \mu \mathrm{m}$ in E-G; 250 $\mu \mathrm{m}$ in inset to $\mathrm{G}$. 

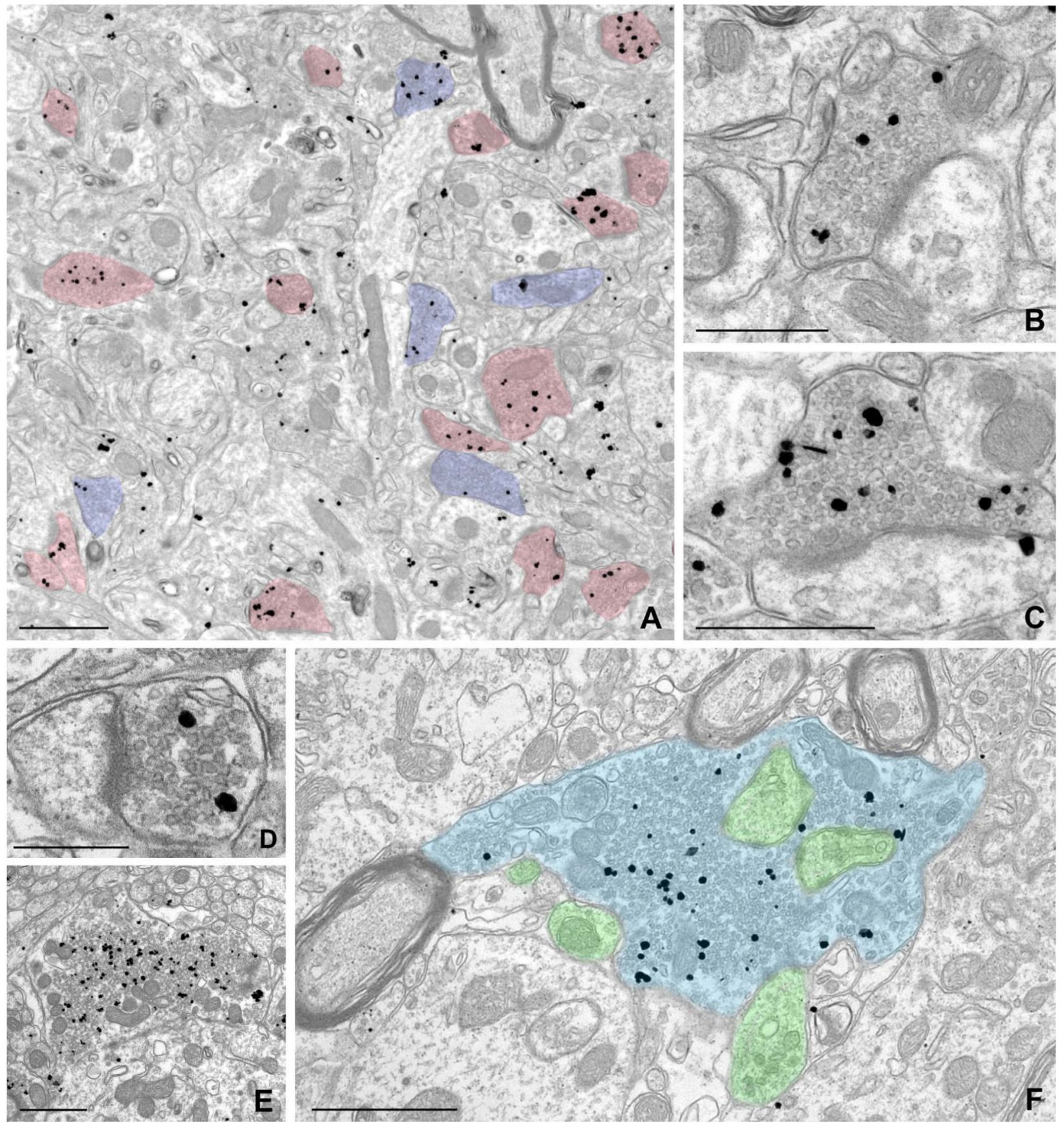

Figure 3.

Pre-embedding immunogold labeling for NDCBE in forebrain. A: Low-magnification electron micrograph from cerebral cortex (layer II/III); labeling for NDCBE concentrates in presynaptic terminals. Terminals with prominent postsynaptic densities are colorized in pink; terminals lacking prominent PSDs and apposed to dendritic shafts are colorized in blue. B,C: Higher magnification views of neocortex, showing labeled presynaptic terminals that make axospinous synaptic contacts. D-F: NBCDE labeling in hippocampus. D: Small terminal in stratum radiatum of CA1. E,F: Large, probable mossy fiber terminals in stratum lucidum of CA3. Terminal in F is colorized blue, and postsynaptic elements are colorized 
green. Note that labeling is associated with the pool of synaptic vesicles, and is excluded from vesicle-poor zones of the terminal. Scale bar $=1 \mu \mathrm{m}$ in A,E,F; $0.5 \mu \mathrm{m}$ in B,C; $250 \mathrm{~nm}$ in $\mathrm{D}$. 

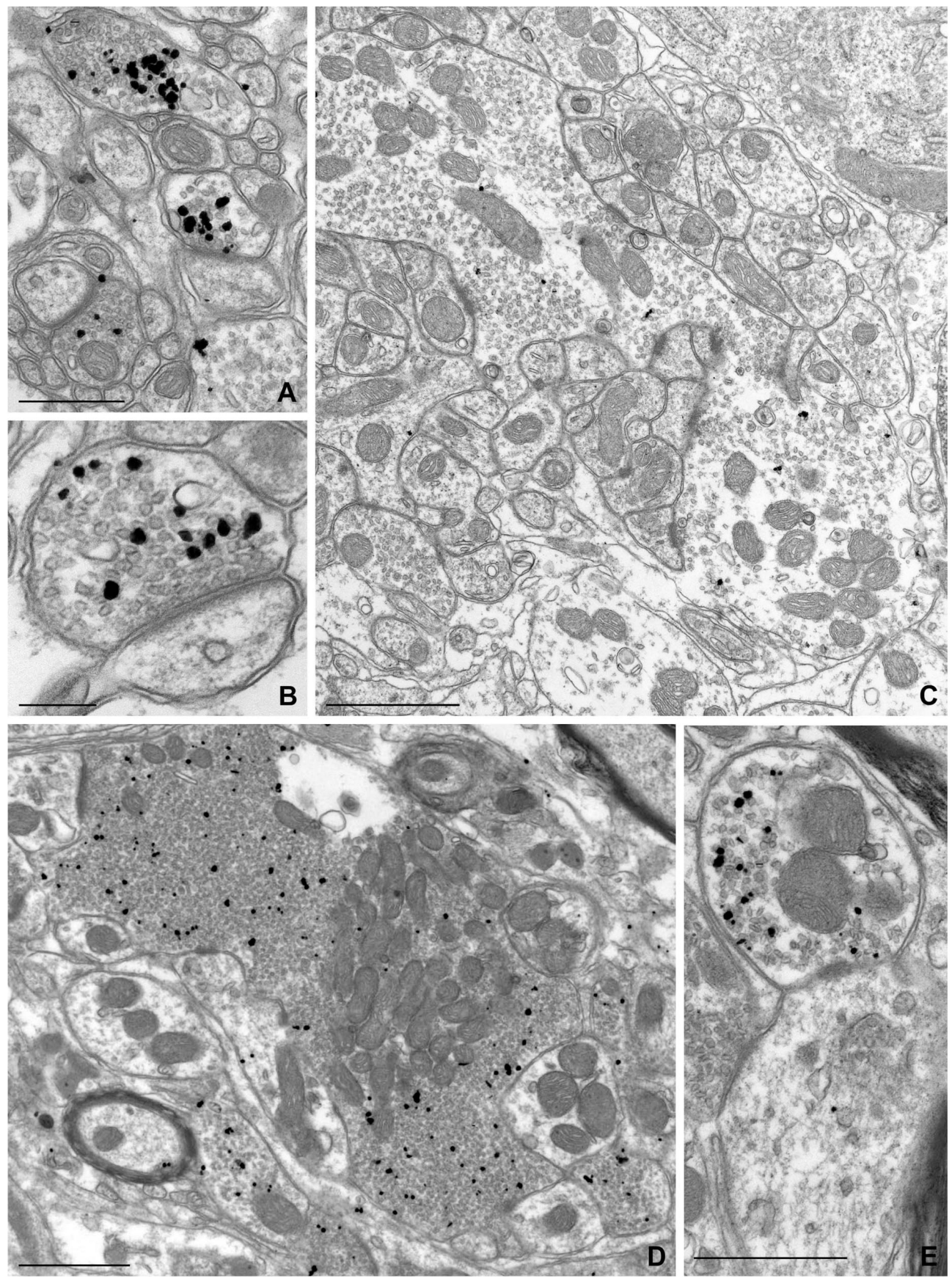

Figure 4.

Pre-embedding immunogold labeling for NDCBE in rat hindbrain. A,B: Labeled terminals in axospinous synapses from the molecular layer of cerebellar cortex. C: Large, weakly labeled terminal in the granule cell layer of cerebellar cortex. D,E: From ventral cochlear nucleus. D: A large presynaptic terminal; labeling is predominantly over zones containing high density of vesicles. E: A small terminal. Scale bar $=500 \mathrm{~nm}$ in A,E; $250 \mathrm{~nm}$ in B; $1 \mu \mathrm{m}$ in C,D. 

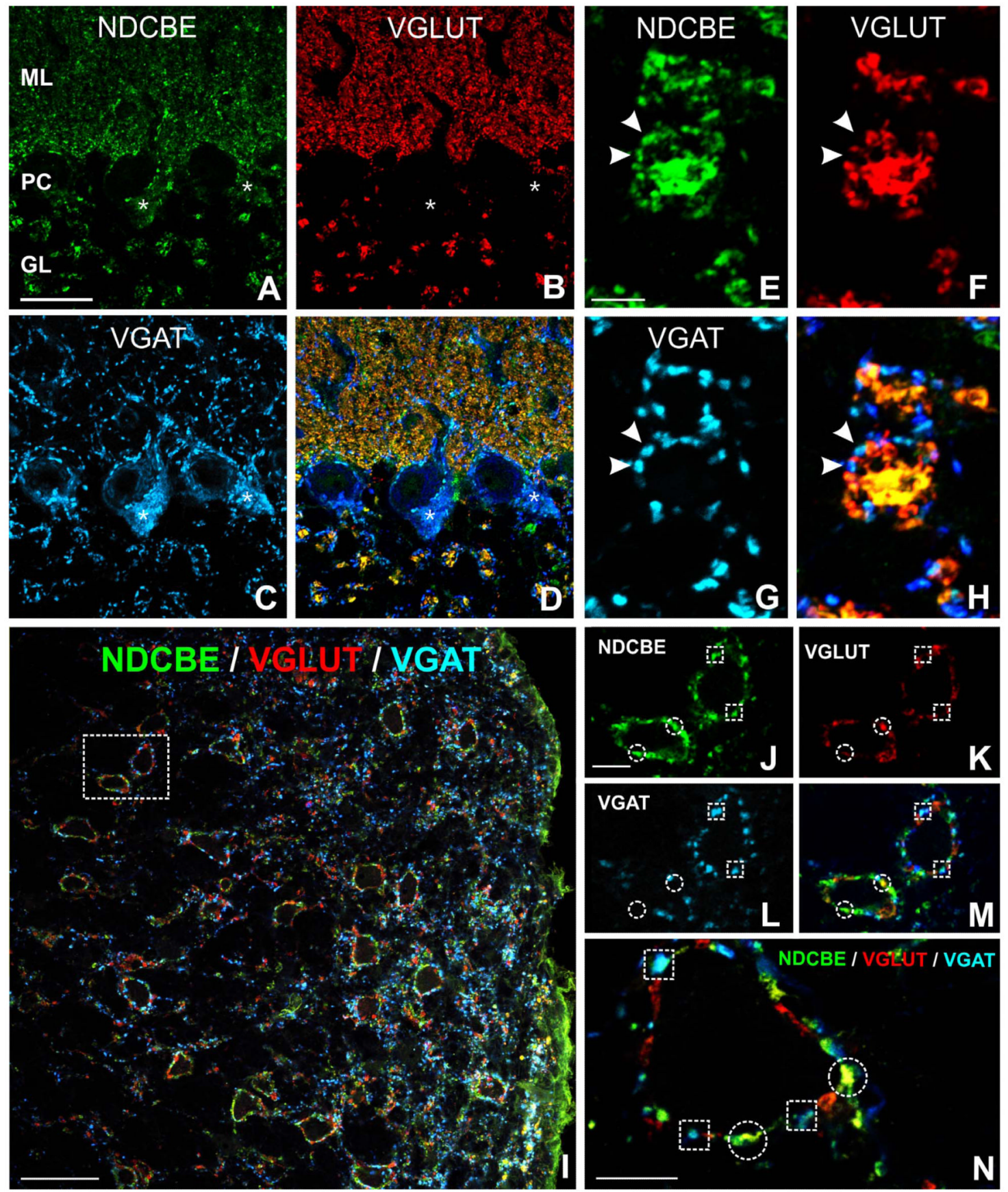

Figure 5.

Co-localization of NDCBE (green) with VGLUT1 (red) and VGAT (blue) in rat hindbrain. A-D: In cerebellar cortex, VGLUT puncta exhibit considerable co-localization with NDCBE; note co-localization between VGAT and NDCBE in the Purkinje cell layer. E-H: Synaptic glomeruli in granule cell layer exhibit a characteristic pattern of co-localization: NDCBE is associated both with the large central VGLUT-positive terminal and with the surrounding VGAT-positive inhibitory terminals. I: Cochlear nucleus. J-N: Co-localization pattern in more detail (J-M are from boxed region in I). Many NDCBE puncta co-localize with VGLUT (circles), whereas others co-localize with VGAT (boxes).Scale bar $=25 \mu \mathrm{m}$ in 
A (applies to A-D); $5 \mu \mathrm{m}$ in $\mathrm{E}$ (applies to e-H); $50 \mu \mathrm{m}$ in I; $10 \mu \mathrm{m}$ in $\mathrm{J}$ (applies to $\mathrm{J}-\mathrm{M}$ ) and $\mathrm{N}$. 

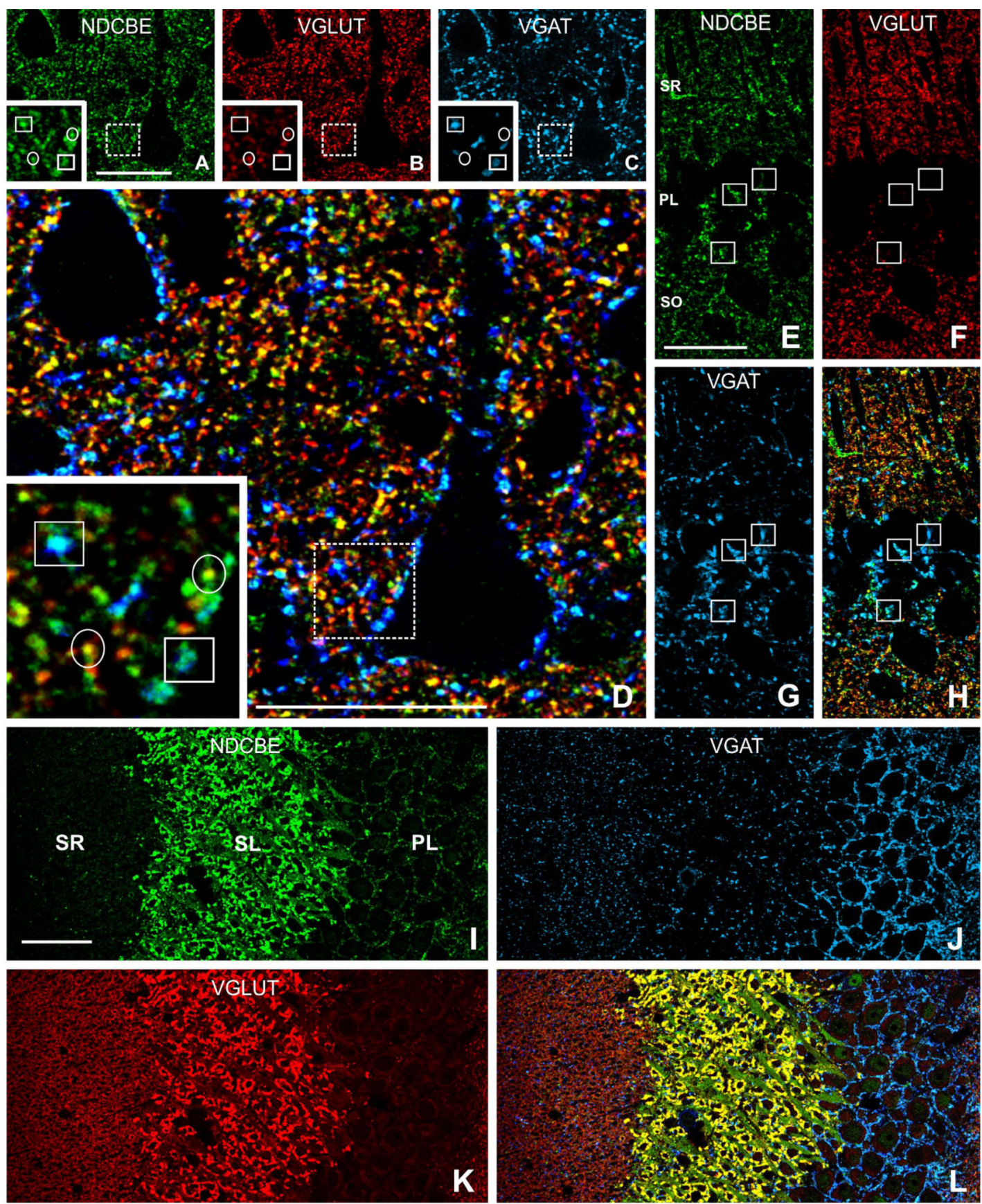

Figure 6.

Triple immunofluorescence for NDCBE (green), VGLUT1 (red), and VGAT (blue). A-D: Many NDCBE puncta in neuropil of cerebral cortex co-localize with VGLUT (circles in insets), whereas others, especially large puncta adjacent to pyramid-shaped somata, colocalize with VGAT (boxes in insets). E-H: Large NDCBE-positive puncta in the pyramidal cell layer of CA1 often co-localize with VGAT (boxes); small yellow puncta in s radiatum $(\mathrm{H})$ indicate co-localization of NDCBE with VGLUT. I-L: CA3 hippocampus; note massive co-localization of NDCBE with VGLUT in stratum lucidum. SL, stratum lucidum; SR, stratum radiatum; PL, stratum pyramidale. Scale bar $=25 \mu \mathrm{m}$ in A (applies to A-C), D, and E (applies to E-H); $50 \mu \mathrm{m}$ in I (applies to I-L). 

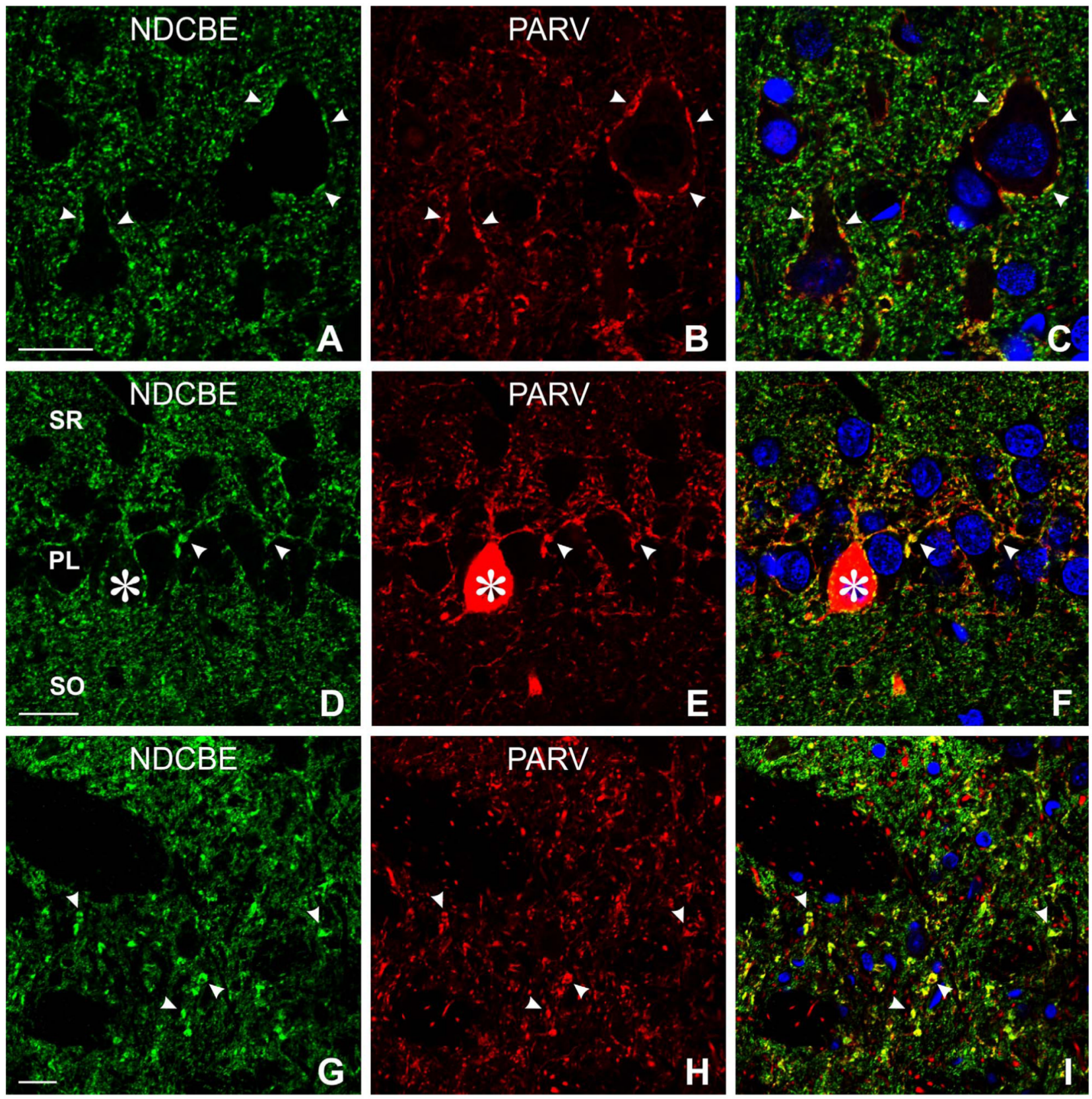

Figure 7.

Double labeling for NDCBE (green) and parvalbumin (PARV, red). A-F: In neocortex (AC), and CA1 hippocampus (D-F), NDCBE co-localizes with PARV in terminal baskets surrounding pyramidal cells. G-I: Thalamus; co-localization is found in large terminals, but these are not organized into baskets. Scale bar $=20 \mu \mathrm{m}$ in A (applies to A-C), D (applies to D-F), and G (applies to G-I). 

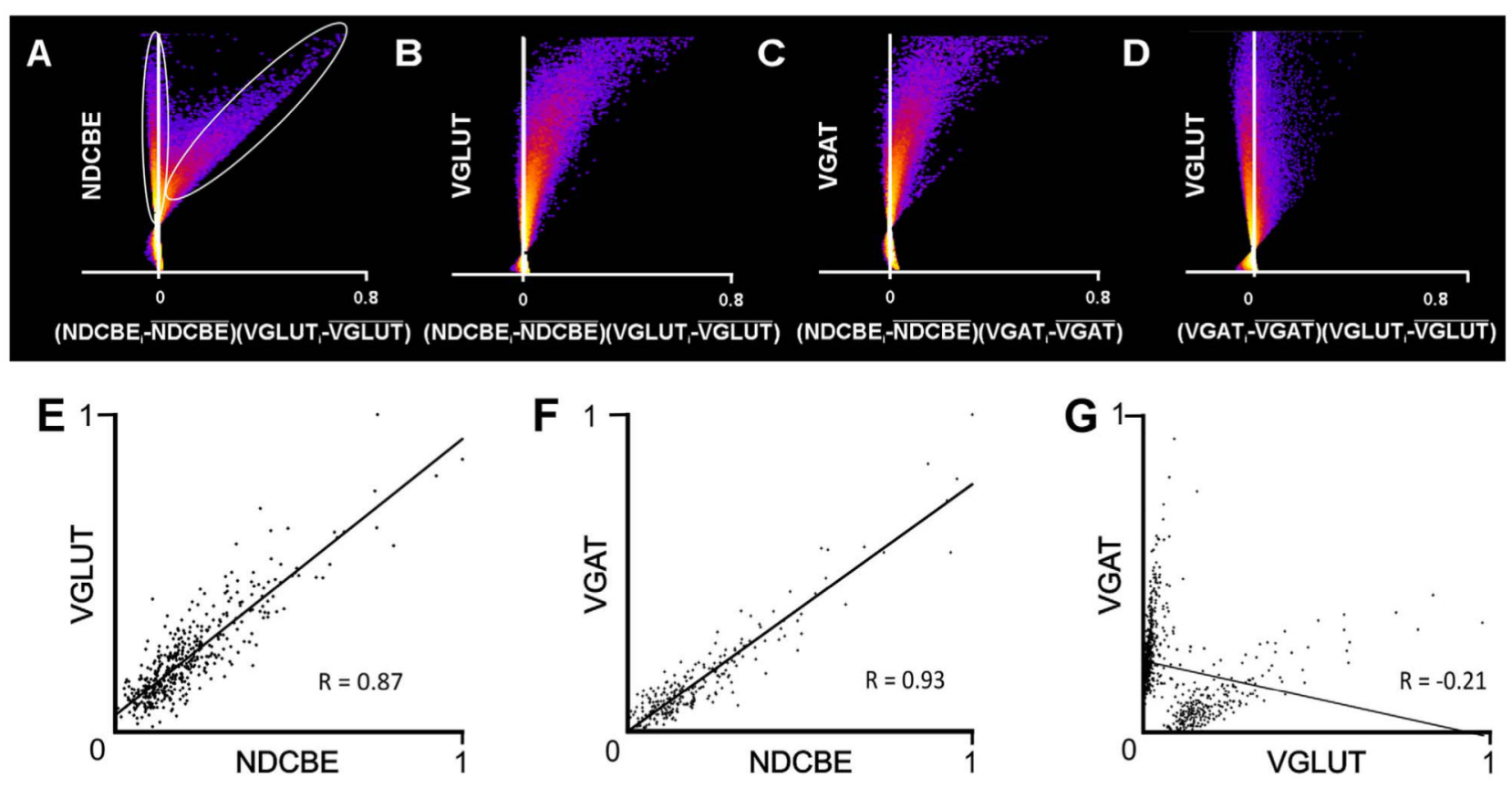

Figure 8.

Scatterplots quantify extent of co-localization. A-D: Intensity correlation plots (designed to assess co-localization for all pixels in a micrograph); "hot" colors represent high density of data points, "cold" colors represent low densities. Dots to the right of the Y-axis indicate positive correlation. The scatterplots are from the raw confocal image used to generate the illustration in Figure 4A. A: Plots NDCBE brightness against VGLUT1 brightness (normalized to its mean value). The data fall into two clouds; the vertical ellipse indicates pixels that exhibit essentially no relationship between NDCBE and VGLUT1, whereas the tilted ellipse indicates pixels that exhibit a strong positive correlation These data suggest that most, but not all NDCBE staining co-localizes with VGLUT1. B,C: Confirmation of substantial co-localization of both VGLUT1 and VGAT with NDCBE. D: In contrast, there is virtually no evidence of co-localization between VGLUT1 and VGAT. E-G: Scatterplots were constructed to define the extent of co-localization within puncta (as defined by both NDCBE and VGLUT1 [E, 443 puncta], both NDCBE and VGAT [F, 254 puncta], or both VGLUT1 and VGAT [G, 692 puncta]). Axes show mean pixel brightness, normalized to run from zero (dimmest punctum) to 1 (brightest). The strong correlation in brightness between the two channels for VGLUT1 and NDCBE (E) and for VGAT and NDCBE (F) contrast with the lack of correlation between $\operatorname{VGLUT1}$ and $\operatorname{VGAT}(\mathrm{G})$. 
TABLE 1

Primary Antibodies Used in This Study

\begin{tabular}{llll}
\hline Primary antibody & \multicolumn{1}{c}{ Immunogen } & Source, species, cat. no. & Dilution \\
\hline NDCBE-1r & C-terminal fragment of rat NDCBE-A/C: & Polyclonal rabbit (reported here) & $1: 2,000$ \\
LSINSGNTKEKSPFN & & Sigma, mouse monoclonal & $1: 40,000$ \\
Parvalbumin & Purified frog muscle parvalbumin & (PARV-19), P-3088 & $1: 1,000$ \\
VGAT & C-terminal peptide of rat VGAT: & $\begin{array}{c}\text { Synaptic Systems, mouse monoclonal } \\
\text { (clone 117G4), 131011. }\end{array}$ \\
VGLUT1 & AEPPVEGDIHYQR & Chemicon, guinea pig polyclonal, AG208 & $1: 5,000$ \\
& C-terminal peptide rat VGLUT1: & & \\
\hline
\end{tabular}

